\title{
Removal of acrylic coatings from works of art by means of nanofluids: understanding the mechanism at the nanoscale
}

\author{
Michele Baglioni, Doris Rengstl, Debora Berti, Massimo Bonini, Rodorico Giorgi and Piero Baglioni $\dagger$ *
}

\author{
Received 14th April 2010, Accepted 25th May 2010 \\ DOI: 10.1039/c0nr00255k
}

Conservation of works of art often involves the inappropriate application of synthetic polymers. We have proposed the use of alternative methodologies for conservation and formulated innovative cleaning nanostructured systems to remove previously applied polymer films and grime from painted surfaces. In particular, a novel "micellar system" composed of water, SDS, 1-pentanol, ethyl acetate and propylene carbonate was recently formulated and successfully used to remove acrylic and vinyl/ acrylic copolymers from Mesoamerican wall paintings in the archeological site of Cholula, Mexico. This contribution reports on the mechanism of the interaction process that takes place between the nanostructured fluid and the polymer coating at the nanoscale. The structural properties of the "micellar solution" and of the polymer film are investigated before, during and after the interaction process using several surface and solution techniques. Rather than a classical detergency mechanism, we demonstrate that micelles act as solvent containers and interact with the polymer film leading to its swelling and detachment from the surface and to its segregation in a liquid droplet, which phaseseparates from the aqueous bulk. After the removal process the micelles become smaller in size and undergo a structural re-arrangement due to the depletion of the organic solvents. These findings can be framed in an interaction mechanism which describes the removal process, opening up new perspectives in the design and formulation of new cleaning systems specifically tailored for intervention on particular conservation issues.

\section{Introduction}

The cleaning of a work of art consists the removal of unwanted materials from its surface. In the past, polymeric films have been applied to coat painted surfaces for protective purposes. However, when the surface of a wall painting is coated with a polymer film (mainly acrylic and vinyl resins), crystallization of salts may take place within the pores of the wall painting itself, leading to strong mechanical stresses inside the painted layer (whose thickness generally is between a few microns and $100 \mu \mathrm{m}$ ) that give rise to the complete disruption of the work of art in a period of time of about few tenths of years, depending on the environmental conditions. In addition to the "support" degradation, the aging of the polymer coating produces drastic changes in the physico-chemical properties of the inorganic constituents of the wall painting ${ }^{1-3}$ and of the polymers themselves $^{4-7}$ (e.g. the permeability to gases, and in particular to water vapor, is highly decreased, the solubility and the mechanical properties of the polymers are strongly reduced, the color of the polymer layer changes during the aging). At the end of the degradation process, these polymers can hardly be removed with neat organic solvents. ${ }^{4}$ Altogether these factors contribute to the complete detachment of the painted layer from the mortar layers.

Micelles and microemulsions are among the most effective systems available for the optimal intervention in these cases, and

Department of Chemistry and CSGI, University of Florence, via della Lastruccia 3-Sesto Fiorentino, 50019 Florence, Italy. E-mail: piero. baglioni@unifi.it; Fax: +39055 525 3032; Tel: +39055 5253033

$\uparrow$ No kinship exists between the authors of this paper. represent an advanced methodology for the cleaning of works of art. $^{8-13}$

The organic phase necessary for the polymer removal is dispersed as nanodroplets in the water continuous phase avoiding the spreading of polymers inside the porous matrix of the artifact. The effectiveness of these soft-nanostructured systems is due to the huge interfacial area that amplifies the interactions with polymer coatings. Moreover aqueous-based microemulsions or micellar solutions largely reduce the toxicity of the application offering at the same time better and more controlled performances than pure organic solvents.

In particular, novel nanofluids have been formulated for the removal of Paraloid B72,9 (ethyl methacrylate/methyl acrylate, 70 : 30 copolymer, supplier Room \& Haas, USA), one of the most used polymers in conservation. An oil-in-water microemulsion containing sodium dodecylsulfate (SDS), 1-pentanol $(1-\mathrm{PeOH})$ as a co-surfactant, and a small amount of $p$-xylene and nitro diluent $\$$ has shown good performances in the removal of this acrylic polymer. However, this system shows some limits in the cleaning of wall paintings where Paraloid B72 and Mowilith DM5 (vinyl acetate/n-butyl acrylate $65: 35$ copolymer, supplier Hoechst, Germany) have been used in combination or sequentially applied to coat the surface of works of art.

This is the case of several Mesoamerican paintings in Mexico, ${ }^{14-16}$ where conservation treatments based on polymer resins

\$ Nitro diluent is a mixture of $62 \%$ toluene, $15 \%$ butyl acetate, $15 \%$ ethyl acetate, $6 \% n$-butyl alcohol, and $2 \%$ cellosolve acetate. The name "nitro diluent" seems misleading since no nitro-derivative is present in the solvent blend. The name comes from the use of the solvent to remove nitro-cellulose based paints, for lacquer dilution, and for cleaning. 

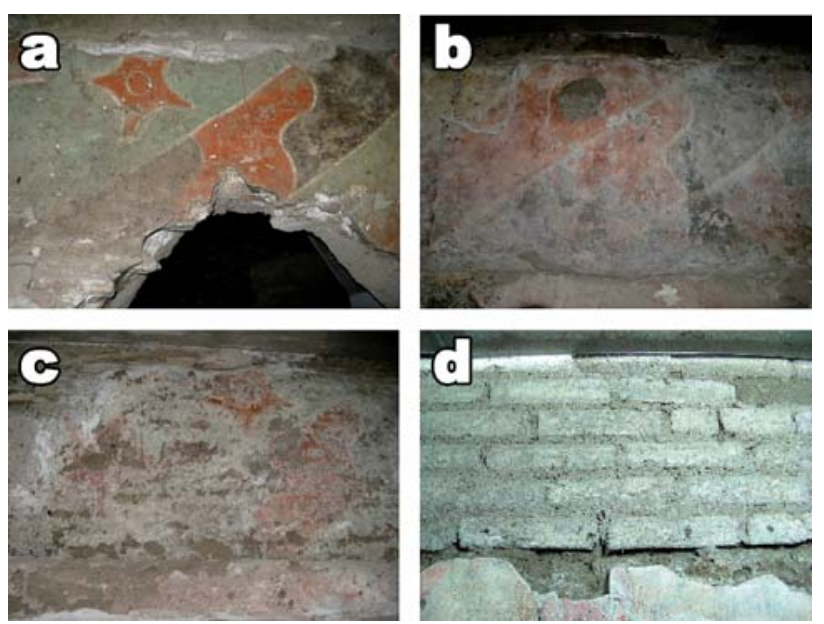

Fig. 1 The picture illustrates (from a to d) the progressing degradative steps of a wall painting in Cholula (Mexico) treated with a combination of acrylic and vinyl polymers. Polymers led to the complete disruption of the painting in a few years after the application.

have contributed in most cases to an irreversible degradation. Recent investigations in the archeological site of Cholula (Mexico, Bebedores de Pulque, 200-300 BC) have shown the dramatic conditions of these paintings, which have been subjected, starting from the end of the sixties, to at least four interventions where polyvinyl acetate/butyl acrylate copolymers (probably Mowilith DM5) and/or Paraloid B72 have been applied sequentially or in mixture as coatings. Most of the paintings are now strongly degraded, due to extensive salt crystallization processes, quickly leading to the disruption of the painted layer and the plaster beneath (see Fig. 1).

This contribution reports on a novel formulation of a sodium dodecylsulfate/1-pentanol, ethyl acetate (EA) and propylene carbonate (PC) (system EAPC) that represents a major advancement in this field. In particular, this system shows a very high effectiveness in removing both Paraloid B72 and Mowilith DM5. Here, we demonstrate how this depends on the specific interactions at the nanoscale between the polymeric coating and the EAPC "micelles". The attention is particularly focused on the interaction between the EAPC system and Paraloid B72.

\section{Experimental section}

\section{Chemicals}

Sodium dodecylsulfate (Sigma-Aldrich, purity $\geq 99 \%$ ), 1-pentanol (Merck, purity $\geq 98.5 \%$ ), ethyl acetate (Sigma-Aldrich, ACS reagents, purity $\geq 99.5 \%$ ), propylene carbonate (SigmaAldrich, purity 99\%) and xylene (Merck, purity $>99.5 \%$ ) were used without further purification. Water was purified with a Millipore MilliRO-6 and MilliQ (Organex Systems) apparatus (resistance $>18 \mathrm{M} \Omega \mathrm{cm}$ ).

For SANS experiments, fully deuterated SDS (Cambridge Isotope Laboratories, Inc., D25, 98\%) and $\mathrm{D}_{2} \mathrm{O}$ (EurisoTop, 98\%) were used as received. Paraloid B72 pellets were purchased from Zecchi, Florence.

\section{Sample preparation}

The EAPC micellar system was prepared starting from a micellar solution of SDS and 1-pentanol in water. Then propylene carbonate and ethyl acetate were slowly added under constant stirring.

The composition of the EAPC system ( $\% \mathrm{w} / \mathrm{w})$ was: water, 73.3\%; SDS, 3.7\%; 1-pentanol, 7\%; ethyl acetate, $8 \%$; and propylene carbonate, $8 \%$. For SANS experiments $\mathrm{D}_{2} \mathrm{O}$ and deuterated SDS were used instead, to enhance the contrast between the "micelles" and the outer solvent phase and minimize incoherent background.

To study the interaction between the EAPC system and Paraloid B72, a 5\% (w/w) Paraloid solution in xylene was laid in glass vials and let dry for at least two days under a gentle flux of nitrogen. The EAPC system was then poured into the vials and these were sealed to avoid solvent evaporation. Four concentrations of the polymer were considered $(1 \mathrm{mg}, 2.5 \mathrm{mg}, 5 \mathrm{mg}$, and $7 \mathrm{mg}$ of polymer per $\mathrm{g}$ of EAPC). The chosen range of concentrations is consistent with the amount of polymer used in restoration of wall paintings and with the amounts of cleaning systems used for the removal. All the samples were kept in a thermostatic bath at $25^{\circ} \mathrm{C}$.

\section{Application tests}

Application tests were performed on laboratory model samples, painted with the fresco technique, that is the application of pigments before the completion of lime hardening. The samples were coated by a Paraloid B72 film applied by brushing the xylene solution. The cleaning was carried out using cellulose pulp poultices imbibed with the AEPC solution and a sheet of Japanese paper interposed between the poultices themselves and the painted surface. The poultices were then covered with a polyethylene film to avoid the fast evaporation of the cleaning liquid systems. After 1.5 hour the poultices were removed and the surface was gently washed with a water-soaked sponge in order to remove possible surfactant residues.

\section{Fourier Transform-Infrared (FT-IR) spectroscopy}

FT-IR measurements were carried out with a BIO-RAD FTS 40PC (Digilab Division) spectrometer. The spectra were recorded as the sum of 32 scans in the $4000-400 \mathrm{~cm}^{-1}$ range with a resolution of $4 \mathrm{~cm}^{-1}$. A DTGS Mid-IR detector was used to collect the signal.

\section{Differential Thermo-Gravimetry (DTG)}

Differential thermo-gravimetry measurements were performed using a SDT Q600 TA Instruments apparatus. The scanned temperature range was $25-300{ }^{\circ} \mathrm{C}$, chosen taking in account the boiling points of the solvents contained in the EAPC system (ethyl acetate, $77^{\circ} \mathrm{C}$; water, $100{ }^{\circ} \mathrm{C}$; 1-pentanol, $138^{\circ} \mathrm{C}$; and propylene carbonate, $240{ }^{\circ} \mathrm{C}$ ). The upper limit of this range coincides with the beginning of the thermal degradation process of Paraloid B72, which is not of interest in this context. The samples $(1-2 \mathrm{mg})$ were put in aluminium open small pans, kept in an inert atmosphere under a nitrogen flux of $100 \mathrm{ml} \mathrm{min}^{-1}$. 


\section{Small-Angle X-ray Scattering (SAXS)}

Small-angle X-ray scattering analyses were carried out with a HECUS SWAX-camera (Kratky) equipped with a positionsensitive detector (OED 50M-1024 channels $54 \mu \mathrm{m}$ wide). $\mathrm{Cu}$ $\mathrm{K} \alpha$ radiation, of wavelength $1.542 \AA$, was provided by a Seifert ID-3003 X-ray generator (sealed-tube type) operating at a maximum power of $2 \mathrm{~kW}$. A $10 \mathrm{~mm}$ thick nickel filter was used to remove the $\mathrm{Cu} \mathrm{K} \beta$ radiation. The sample-to-detector distance was $274 \mathrm{~mm}$. The volume between the sample and the detector was kept under vacuum during the measurements to minimize the scattering from the air. The temperature during the experiment was set to $25^{\circ} \mathrm{C}$. The Kratky camera was calibrated in the small angle region using silver behenate $(d=58.38 \AA) .{ }^{17}$ The samples were contained in $1.5 \mathrm{~mm}$ thick quartz capillary tubes sealed with hot melting glue.

\section{Quasi-Elastic Light Scattering (QELS)}

Quasi-elastic light scattering experiments were carried out at a temperature of $25^{\circ} \mathrm{C}$ with a Brookhaven Instrument apparatus (BI9000AT correlator and BI $200 \mathrm{SM}$ goniometer). The light scattered from the sample has been collected at $90^{\circ}$ with respect to the incident $532 \mathrm{~nm}$ laser light radiation (the double frequency of a pumped Nd:YAG diode from Coherent-Compass $315 \mathrm{M}$, whose power output was $100 \mathrm{~mW}$ ). The samples were contained in sealed glass light scattering tubes.

\section{Small-Angle Neutron Scattering (SANS)}

Small-angle neutron scattering measurements were performed on the PAXE spectrometer of the Laboratoire Léon Brillouin, LLB (Saclay, Gif-Sur-Yvette, France), using two different configurations, either for the high or the low $Q$ range $(Q=(4 \pi / \lambda) \sin (\theta / 2)$, where $\lambda$ is the wavelength of the incident neutron beam and $\theta$ is the scattering angle). In the high $Q$ configuration an average wavelength of $5 \AA$ was used, with a sample-to-detector distance of $1.8 \mathrm{~m}$. In the low $Q$ configuration an average wavelength of 11 $\AA$ was used, with a sample-to-detector distance of $5 \mathrm{~m}$. It is worth noting that the two $Q$ ranges partially overlapped, so that it was easy to merge the two curves obtained. The wavelength spread, $\Delta \lambda / \lambda$, was for both cases less than $10 \%$. The overall $Q$ range investigated for all samples was $0.017<Q<0.256 \AA^{-1}$. Scattered neutrons were detected by a two dimensional $X Y$ position detector with $64 \times 64$ active elements $\left(\mathrm{BF}_{3}\right)$ covering a total area of $4096 \mathrm{~cm}^{2}$. Samples were contained in $1 \mathrm{~mm}$ thick quartz cells and they were kept at $25{ }^{\circ} \mathrm{C}$ during the measurements. The scattering intensity was corrected for the empty beam contribution, transmission, and detector efficiency and it was normalized to the absolute scale by a direct measurement of the intensity of the incident neutron beam. The integration of the normalized 2D intensity distribution with respect to the azimuthal angle yielded the $1 \mathrm{D}$ scattering intensity distribution, $I(Q)$ in $\mathrm{cm}^{-1}$. The reduction of the data was performed using standard routines available at the LLB.

The micelles were modeled as charged prolate ellipsoidal particles interacting with each other according to a screened Coulomb potential described by the NAR-MMSA (non-additive radius multi-component mean sphere approximation) model. To fit the data, already normalized to absolute scale, a modified routine (NCNR_SANS_package_6.011 available from NISTNational Institute for Standard and Technology, Gaithersburg, USA) running on Igor Pro (Version 6.05A) was used.

\section{Scanning Electron Microscopy (SEM)}

SEM investigations were carried out with a Cambridge Stereoscan 360 working at $20 \mathrm{kV}$ of acceleration potential and $25 \mathrm{~mm}$ of working distance. Small samples were taken out of the surface of the fresco model sample with the help of a scalpel and then coated with a gold layer using an Agar Scientific Auto Sputter Coater 108A.

\section{Atomic Force Microscopy (AFM)}

AFM measurements were carried out with a PSIA XE-100E system. Drop-cast samples were prepared by applying a droplet of a $30 \%(w / w)$ solution of Paraloid B72 in xylene on top of freshly cleaved mica substrates. The samples were then let slowly evaporate by placing on top of them a round clock glass turned upside down.

A known amount of the EAPC system (i.e. $200 \mu \mathrm{l}$ ) was laid on the treated mica slides and let interact for 10 seconds. To stop the interaction process the mica slides were quickly immersed in a large amount of water that diluted the EAPC system and interrupted the polymer removal. The samples were then let dry for a few days and observed on the AFM instrument. All the images were acquired in non-contact mode by using a silicon cantilever (force constant, $42 \mathrm{~N} \mathrm{~m}^{-1}$ ) with a silicon nitride tip (radius of curvature $<5 \mathrm{~nm}$ ).

\section{Results and discussion}

The use of neat solvents for the removal of grime or unwanted organic materials applied to works of art is common practice in conservation. Although organic solvents can remove most of the materials deposited on the surface of the artifact, their use is unpractical in cleaning porous materials due to the re-deposition of the polymers inside the pores and on the surface of the artifacts. In previous studies we have shown that nanostructured fluids are very efficient in removing grime and polymers. ${ }^{8-13,18,19}$ Ethyl acetate and propylene carbonate can be used to remove polymer contaminants since they are able to dissolve Paraloid B72. However, saturated aqueous solutions of EA or PC $(\mathrm{EA} \approx 8 \% \mathrm{w} / \mathrm{w}$, $\mathrm{PC} \approx 20 \% \mathrm{w} / \mathrm{w}$ ), or micellar solutions with SDS and 1-PeOH only are ineffective in polymer removal and provide only partial swelling of the film. The addition of ethyl acetate and propylene carbonate to a sodium dodecylsulfate and 1-pentanol micellar solution, in the stoichiometric ratios reported in the Experimental section (EAPC nanofluid), boosts the removal process, resulting in the full disappearance of the polymeric coating from the surface and from the porous texture of the painted layer.

The presence of "micelles" is therefore required, but not sufficient, to obtain good performances. Moreover, without SDS, the solvent mixture undergoes phase separation due to the low water solubility of 1-PeOH and ethyl acetate.

Some application tests on a model sample, simulating a real fresco wall painting, have been performed. The EAPC formulation provides a complete cleaning of the painted surface treated with Paraloid B72 in a few hours. Removal is here referred to the 
combined action of swelling, solubilization, and detachment of the polymer film from the painted surface.

SEM images (see Fig. 2) show the appearance of a wall painting model sample before (a) and after (b) the application of a Paraloid B72 coating. Picture (c) shows the surface after the removal of Paraloid B72 by using the EAPC system. It is worth noting that the application of the previously mentioned aqueous saturated solution of organic solvents without SDS (d) results in a swelling of the polymer layer without any appreciable removal of the coating. From what observed, it is clear that the synergistic action of micelles, PC and EA co-solvents is the key of the cleaning effectiveness of the system.

The microscopic observation substantially confirms what is visible to the naked eye, i.e. the removal of the Paraloid B72 film. While this is already sufficient for practical applications, it is clear that the design of efficient formulations tailored for the removal of a given polymeric coating requires a deep comprehension of the solubilization mechanism, that necessarily involves both a structural characterization of the nanofluid and of the interaction mechanism. This characterization should be clearly performed at the nanoscale resolution.

With this in mind, some removal experiments have been performed on polymer films deposited at the bottom of glass test tubes through evaporation of their organic solutions. A known amount of the EAPC system has been added into the vial where the polymeric film was previously laid. The swelling and the detachment of the polymer film can be visually observed in a very short time (typically a few tenths of minutes). The macroscopic observed effect is a phase separation, from a solid-liquid system (polymer film/EAPC) to a liquid-liquid biphasic system, where the polymer becomes confined in a viscous low-density fluid, which separates as a nearly spherical droplet floating onto the aqueous phase.

Fig. 3 reports the removal process and the subsequent phase separation. Hereafter we will refer to the higher density waterrich phase as "lower phase" and to the lower density phase as "upper phase" or "drop-like" phase to stress its unusual globular appearance with respect to regular Winsor I systems. ${ }^{20}$
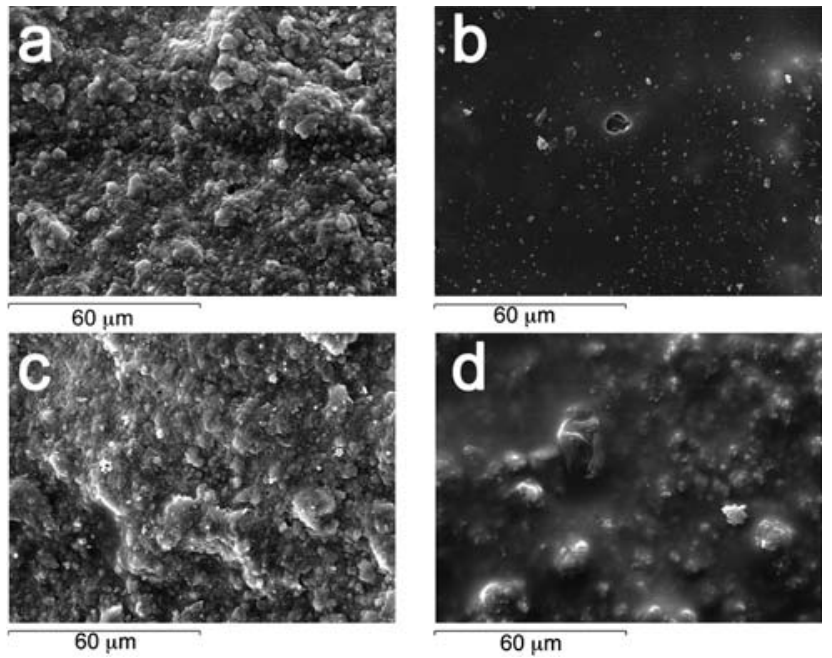

Fig. 2 SEM micrographs of the sample reproducing a wall painted with the fresco technique.
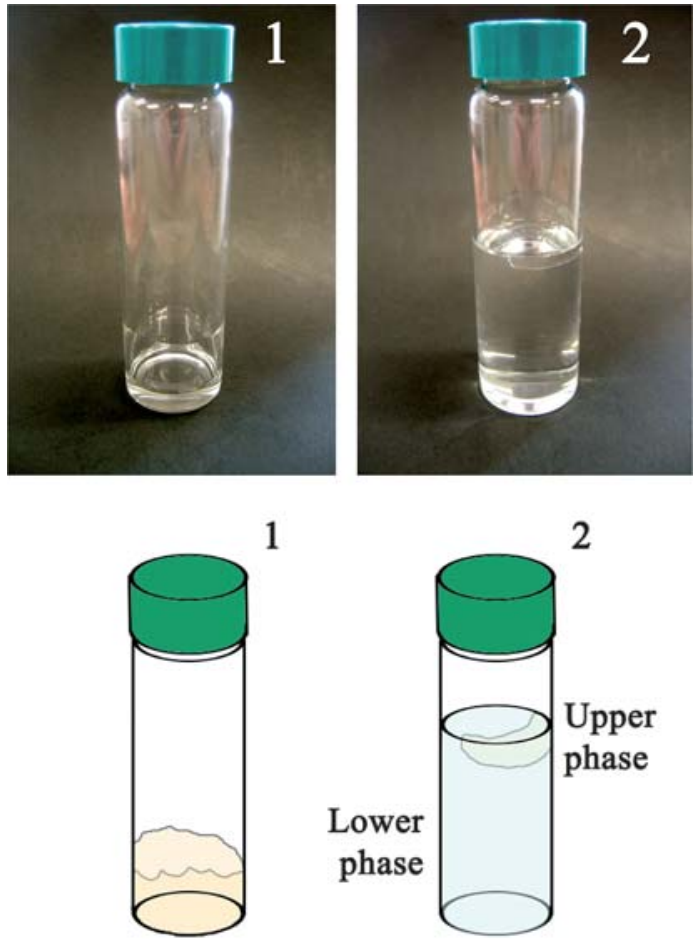

Fig. 3 Visual appearance of the result of tests performed in glass tubes. The schematic cartoon helps in understanding the pictures. Two liquid phases resulting from the interaction process between the micellar system and the polymer are clearly observable. (1) Vial with polymer only, before the interaction with the EAPC system. (2) Vial at the equilibrium after the interaction between the polymer coating and the EAPC micelles.

In the following sections we report the characterization of the composition of the two liquid phases, i.e. the phase structure at the nanoscale, the composition before and after the removal process and what happens during the removal process.

FT-IR measurements were performed in order to determine the partition of Paraloid B72 between the two phases. Fig. 4 shows the FT-IR spectra of the upper and lower phases, and of Paraloid B72. A qualitative inspection clearly indicates that the lower water-rich phase does not contain appreciable quantities of

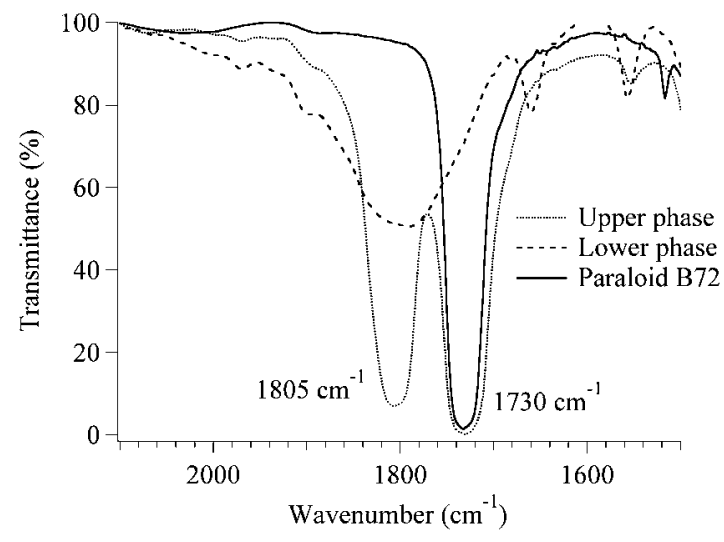

Fig. 4 FT-IR spectra of the upper and lower phase and of Paraloid B72. Here is clearly visible that the polymer is only included in the upper phase. 
Paraloid B72 while the polymer is certainly present in the upper phase, as deduced from the comparison of the spectra in correspondence of the absorption at $1730 \mathrm{~cm}^{-1}$ (esteric $\nu \mathrm{C}=\mathrm{O}$ stretching of Paraloid B72). The peak at $1805 \mathrm{~cm}^{-1}$ present in the lower and upper phases is due to the $\nu \mathrm{C}=\mathrm{O}$ stretching of propylene carbonate, which was not completely evaporated from the samples (the boiling temperature for this solvent is $240{ }^{\circ} \mathrm{C}$ ).

A more quantitative picture of the composition of the upper phase can be inferred from a DTG analysis, which was feasible thanks to the different boiling points of these solvents, relatively spaced in the temperature axis.

\section{Composition of the upper phase}

Differential thermo-gravimetry (DTG) was used to characterize the polymer-rich drop-like phase. Fig. 5 reports the first derivative of the weight loss versus a temperature ramp measured on three different samples. Curve A was recorded on a small amount of the upper phase coming from a sample containing $1 \mathrm{mg}$ of Paraloid B72 per gram of EAPC micellar solution. Curve B is the same as above, but the analyzed mixture contained $7 \mathrm{mg}$ of polymer per gram of EAPC. Finally, curve $\mathrm{C}$ is a solution of Paraloid B72 in a blend of the solvents used in the formulation, selected after several attempts to fit the real profile. The composition of the reconstructed blend is the following $(\% \mathrm{w} / \mathrm{w})$ : Paraloid B72, 25\%; EA, 32\%; 1-PeOH, 23\%; PC, 14\%; and water, $6 \%$. It is clearly visible that this blend is particularly enriched in the less polar components of the micellar system.

The quantitative compositional analysis of the drop-like phase reveals more details about the specific behavior of the polymer respect to the organic solvents blend used in the formulation.

In particular, it is interesting to notice that the composition of the drop-like phase is practically invariant (the shapes of curves A and B are very similar), independently from the amount of

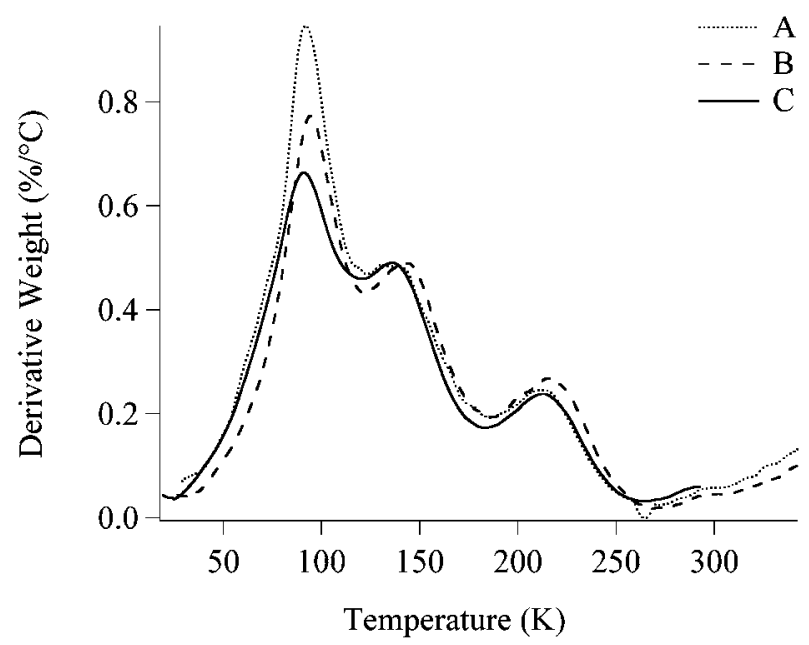

Fig. 5 Comparison between three different DTG thermograms. A (dotted line), upper phase coming from a sample containing $1 \mathrm{mg}$ of Paraloid B72 per gram of EAPC; B (dashed line), upper phase coming from a sample containing $7 \mathrm{mg}$ of Paraloid B72 per gram of EAPC; C (solid line), simulated upper phase, reconstructed dissolving $25 \%(\mathrm{w} / \mathrm{w})$ of Paraloid B72 in a mixture of ethyl acetate (32\%), 1-pentanol $(23 \%)$, propylene carbonate $(14 \%)$ and water $(6 \%)$.

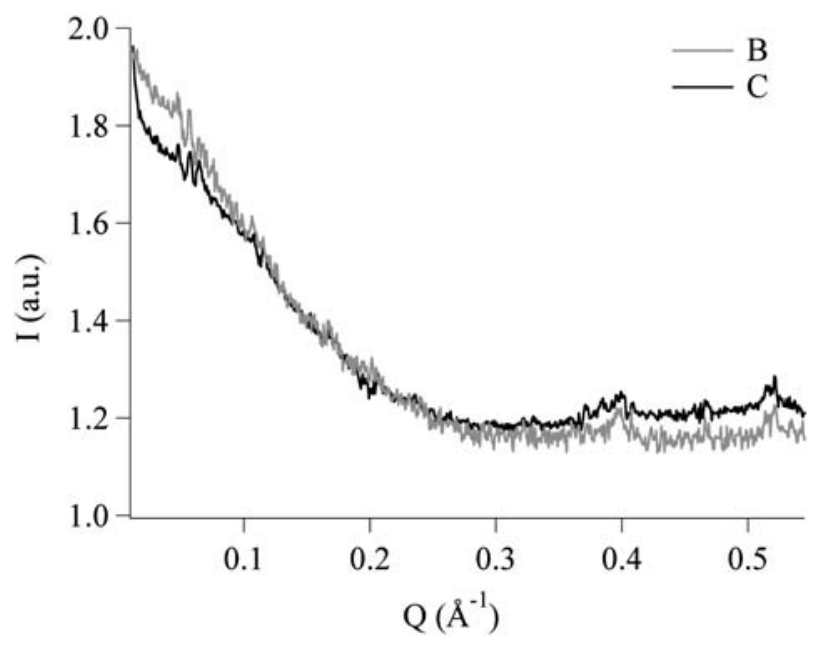

Fig. 6 SAXS curves of two of the same samples analyzed in DTG. The very good matching between the two scattering patterns confirms that the reconstructed upper phase is a good simulation of the real one.

Paraloid B72 added to the same quantity of the micellar solution. In other words, the variation in the initial polymer quantity only affects the volume of the upper phase and not its composition.

This suggests that the polymer "chooses" the optimal amounts of solvents to undergo a swelling process and, therefore, the cleaning system must fulfill specific compositional requirements to get good performances.

As a further confirmation of this hypothesis, SAXS curves were also recorded on $\mathrm{B}$ and $\mathrm{C}$ samples. The scattering patterns (see Fig. 6) show a good matching, as in DTG, between the real upper phase and the simulated mixture.

A simple and straightforward analysis of the SAXS spectra is precluded by the inter-chain correlations (i.e. the excluded volume) or possibly entanglement of the polymeric chains, due to the high concentration of the polymer. For this reasons only a simple qualitative and comparative analysis of the scattering pattern has been performed.

In line with this assumption, it is also possible to infer the bulk composition of the lower phase, which results to be $(\% \mathrm{w} / \mathrm{w})$ water, $74.3 \%$; SDS, 3.8\%; 1-pentanol, 6.6\%; propylene carbonate, $7.8 \%$; and ethyl acetate, $7.5 \%$.

According to these results, the upper phase is a macromolecular solution while the lower phase should still be nanostructured. Therefore the bulk composition is not particularly informative, unless the micro-structural properties and composition at the nanoscale are known.

\section{Characterization of the lower phase}

Complex amphiphiles based nanofluids can be conveniently characterized in terms of structural properties by Small-Angle Scattering Techniques. ${ }^{21}$

Small-Angle Neutron Scattering is particularly suited for multi-component systems, because it lends itself to H/D isotopic substitution, which causes external/internal contrast variation. Different components and/or regions of the aggregates can be spotted and isolated from the background, virtually not changing the chemical composition and properties of the system. 
We have performed a series of SANS measurements with contrast variation to determine the composition of EAPC. ${ }^{22}$

We should specify here that we use the term "micelle" to indicate aggregates composed of a surfactant (SDS) a cosurfactant $(1-\mathrm{PeOH})$ and the two co-solvents (EA and PC), which are solvents with partial water miscibility. Generally speaking, this is not a "classical micellar solution", but it is not even a "classical microemulsive" system, where an immiscible solvent is dispersed in water separated by a surfactant layer. Therefore we use the term micelle or swollen micelle to indicate this nanocompartment.

The SANS spectrum of EAPC has been compared to the pattern of the lower phase, which is what remains after the interaction with the polymer, as shown in Fig. 7.

The scattering data have been interpreted assuming for the micelles a monodisperse homogeneous prolate ellipsoidal form factor. Due to the complexity of this five-components system a simple two-contrast model (micelle-solvent) was used, where each scattering length density (SLD) is consistent with the composition of the scattering objects.

The deviation from a globular shape for such micelles is justified in view of the classical studies on SDS/1-PeOH ${ }^{23-26}$ that point out an axial growth when the co-surfactant is added to the amphiphilic solution.

The main feature of both spectra is the presence of a pronounced inter-particle correlation peak, due to the Coulombian repulsion between the charged micelles. This correlation has been introduced in the model as due to an inter-particle screened repulsive potential. ${ }^{27}$ The peak position is related to a mean inter-particle correlation distance in the real space: $Q_{\max }=2 \pi / d$.

If we compare the spectra, we notice at first sight a more than two-fold drop in scattered intensity of the lower phase after phase separation with respect to AEPC. Moreover the position of the correlation peak is shifted to a higher scattering vector $Q$. The mean inter-micellar distance is about $112 \AA$ for EAPC and $84 \AA$ for the lower phase after polymer extraction.

The scattered intensity is given by:

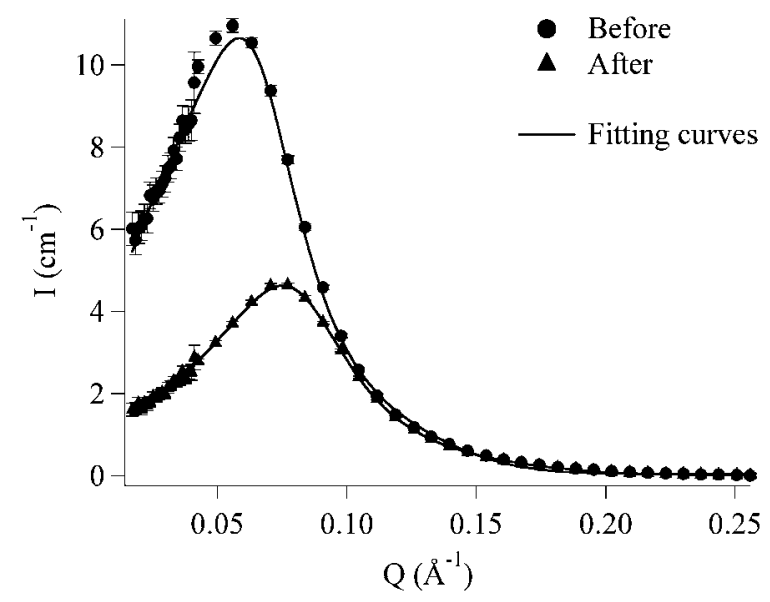

Fig. 7 SANS curves of the EAPC system (circles) and of the lower phase (triangles). The experimental data are fitted (solid lines) according to the fitting model described in the text.

$$
I(Q)=N_{\mathrm{p}} V^{2} \Delta \rho^{2} P(Q) S(Q)
$$

where $P(Q)$ is the form factor, accounting in our case for the ellipsoidal shape of the micelles, $S(Q)$ is the structure factor, $N_{\mathrm{p}}$ is the number density of scattering objects, $V$ is their volume and $\Delta \rho$ is the contrast term, which is the squared difference of the scattering length densities between the micelles and the solvent.

The micelles have an effective charge $Z$ and are described by the following geometrical parameters: $a$ (major semi-axis) and $b$ (minor semi-axis) sufficient to identify a prolate ellipsoid. The other three parameters that complete the fitting model are the scattering length densities (SLDs), $\rho_{\text {mic }}$ and $\rho_{\text {solv }}$, that describe the contrast between the micelle and the solvent and the volume fraction, $\Phi$, of the scattering objects with respect to the whole system volume.

Three out of the six fitting parameters (i.e. $\rho_{\text {mic }}, \rho_{\text {solv }}$, and $\Phi$ ) were considered to be semi-fixed and constrained in a small range of likely values. The remaining three parameters (i.e. $a, b$, and $Z$ ) were the output of the fitting and they were let free to change their values (see Table 1).

The reason for the intensity decrease can be the combination of a shrinking of the micelles and/or a decrease of the contrast term. In the present case, we know from DTG analysis that the aqueous phase is depleted of the less polar solvents and, particularly, ethyl acetate and $1-\mathrm{PeOH}$ are extracted to the upper phase. We expect, from the known scattering lengths of the components, a drop in the contrast term. However, this is not the only effect, as the simultaneous shift of the correlation peak reveals, suggesting a concomitant decrease of the micellar size. The model fitting above introduced can address this point. EAPC micelles have a radius of $20.8 \AA$ and an axial ratio of 4.3 , while the micelles in the lower phase display a radius of $18.0 \AA$ and an axial ratio of 3.7 .

To summarize, polymer extraction and phase separation leave in the lower phase a nanostructured fluid still composed of micelles, which are now smaller, due to the depletion in organic solvents.

This conclusion in turn confirms the hypothesis that SDS/1$\mathrm{PeOH}$ nanostructures contain a certain amount of the cosolvents because size reduction is a direct consequence of the outflow of the organic solvents that leave the micellar phase to dissolve the polymer.

QELS curves (see Fig. 8) were also collected after the interaction of different Paraloid B72 amounts with the same quantity of the EAPC system. It can be noticed (see inset in Fig. 8) that the micelle size decreases with Paraloid B72 content increase. This is consistent with what previously stated: if the concentration of the polymer is raised up, the polymer itself needs more and more

Table 1 The SANS fitting parameters

\begin{tabular}{lll}
\hline Fitting parameters & EAPC & Lower phase \\
\hline$\Phi$ & 0.18 & 0.20 \\
$a / \AA$ & $89.1 \pm 0.6$ & $65.9 \pm 0.2$ \\
$b / \AA$ & $20.8 \pm 0.1$ & $18.0 \pm 0.1$ \\
$\rho_{\text {mid }} / \AA^{-2}$ & $1.86 \times 10^{-6}$ & $2.35 \times 10^{-6}$ \\
$\rho_{\text {solv }} / \AA^{-2}$ & $5.55 \times 10^{-6}$ & $5.50 \times 10^{-6}$ \\
$Z\left(1.60 \times 10^{-19} \mathrm{C}\right)$ & $6.4 \pm 0.1$ & $8.3 \pm 0.1$ \\
\hline
\end{tabular}




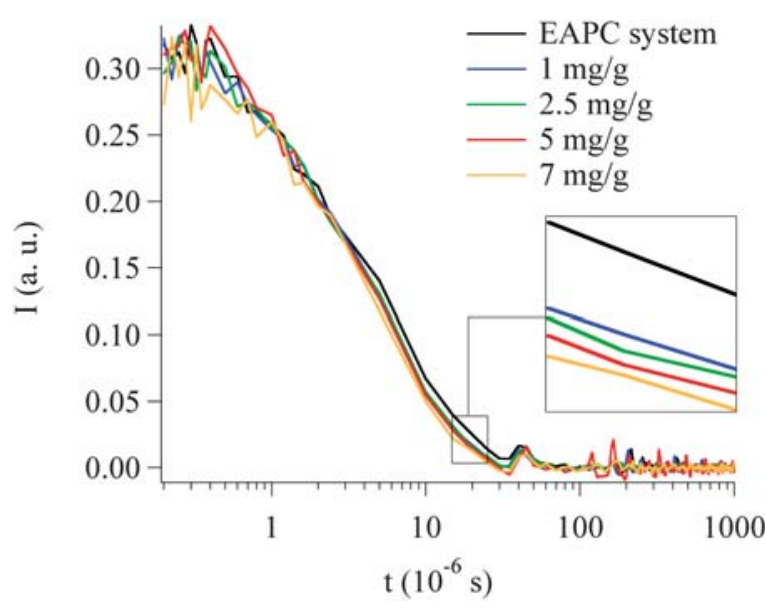

Fig. 8 QELS curves acquired on EAPC samples containing different concentration of Paraloid B72. The measurements were carried out on the lower phase of each sample.

solvent mixture to maintain constant the composition of the blend where it solubilizes in.

\section{AFM: insights on the mechanism of cleaning}

The cleaning process is the result of many different phenomena that take place either at the same time or sequentially. The polymer coating has to be first swollen by a "good" solvent mixture and then it should be detached from the surface, and finally dispersed in the liquid phase to avoid a re-deposition. The higher is the efficiency of each step, the better is the cleaning process. Nevertheless, the highest efficiency is not the only important parameter. In the specific case of removal of polymer coatings from the surface of works of art, all these processes must happen in a highly controlled and sequential manner. The wetting and the removal of the polymer film should be homogeneous to produce the best results from an esthetic point of view, minimizing the wetting of the surface below the polymer film (with no clean areas directly exposed to the swelling solvents while the film has still to be removed in some other areas), and avoiding any possible damage to the original artwork.
It is therefore very important to know the mechanism through which the cleaning process takes place. To this end it is crucial to directly investigate the surface during the cleaning.

Atomic Force Microscopy is a well-suited technique to investigate surface phenomena with nanoscale resolution and then unravel the processes contributing to the mechanism of cleaning. ${ }^{28}$ As a model system we have chosen to drop-cast the film on top of an atomically flat mica substrate to avoid any effects arising from the roughness of the support. In this approach the roughness of the polymer film is only due to the intrinsic properties of the polymer itself and to the preparation protocol.

In real systems the different roughness between, for instance, oil paintings and frescoes is so large that it would definitely play a major role in the cleaning process. However, a detailed investigation on the effects of roughness goes beyond the scope of this work.

AFM experiments were performed in order to investigate the cleaning mechanism at the nanoscale, avoiding any effect arising from the morphology of the substrate. In this framework, it is important to point out that the experiments performed by SEM and AFM are complementary, because they tackle two different aspects of the cleaning process. The sample investigated by SEM is a portion of a real fresco, where Paraloid has been applied with a conventional brush. This experiment is therefore directly related to the visual appearance of a real artwork, whose roughness makes the AFM investigation not even feasible. On the other hand, AFM experiments were carried out on "model samples" with unrealistically low roughness and polymer film thickness, to highlight the individual processes contributing to the cleaning process.

In Fig. 9a an optical image of the polymer film on mica is reported. No significant features could be detected, indicating that the film is homogeneous from the millimetre down to the micron scale. In Fig. 9b a representative AFM micrograph of the polymer film is shown, together with the height profile along three lines (Fig. 9c). The film is quite homogeneous, with a maximum peak to valley roughness of about 3 nanometres (see red line). Sub-micrometric voids and crystals are also present, covering less than $5 \%$ of the total area.

To investigate the cleaning action of the EAPC system, a known volume $(200 \mu \mathrm{l})$ of the system was added on top of the
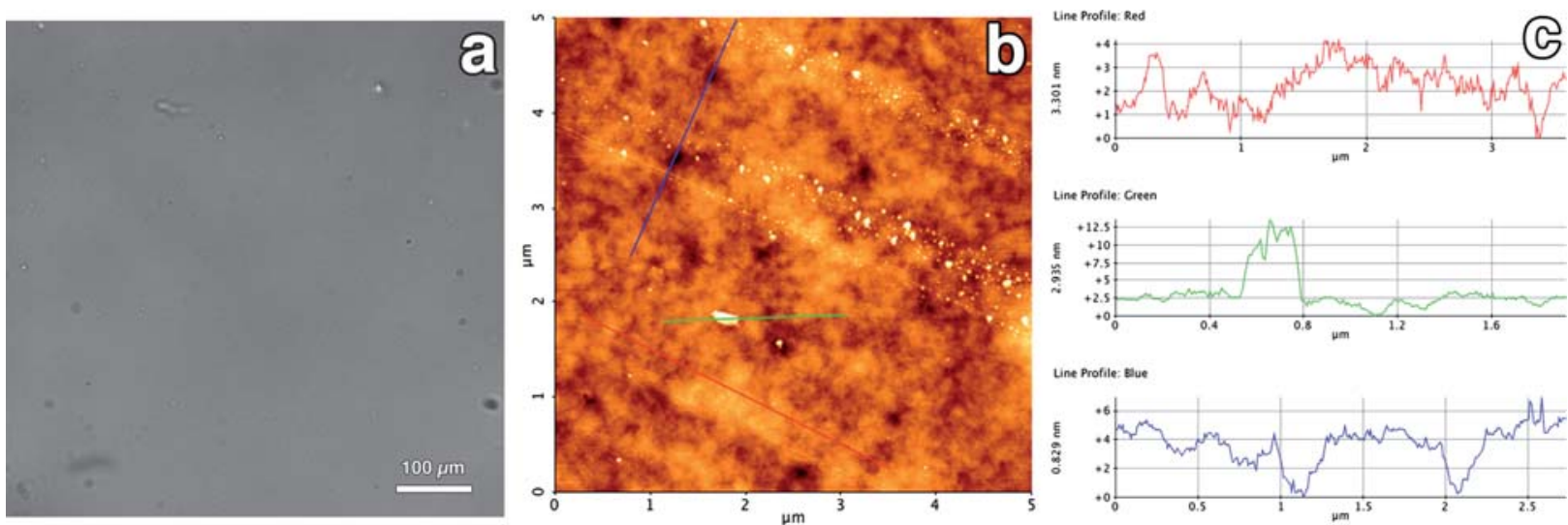

Fig. 9 Optical (a) and AFM (b) micrograph (height range: $13 \mathrm{~nm}$ ) of the Paraloid film as obtained by drop casting on a mica slide. The height profile along three lines is also reported (c). 
polymer-coated mica slide $\left(2.5 \times 2.5 \mathrm{~cm}^{2}\right)$. The role of SDS in the cleaning was evaluated by using a mixture identical to the previously described EAPC system but without SDS. Since this mixture is a biphasic system, after vigorous shaking and phase separation, the saturated aqueous phase has been applied. In all the experiments, the slide was plunged in water after 10 seconds of treatment to quench the polymer removal (Paraloid is insoluble in water). The samples were then left to dry under a hood, observed by optical microscopy and then investigated by means of AFM in non-contact mode.

Fig. 10a shows an optical image of the polymer film treated with the EAPC system without SDS. The presence of cleaned areas together with regions where the film is still present can be detected already by naked eye. In the optical micrograph the border between the clean region (on the left) and the polymer film (on the right) is clearly visible and indicated by a white arrow. Furthermore, globular aggregates and fibrillar structures, not originally present in the polymer film before the treatment, are visible. These results indicate that the uncontrolled wetting arising from EAPC system without SDS leads to the removal of Paraloid in millimetre scaled regions, while other regions are still covered by the polymer. Further, the absence of SDS results in the re-deposition of part of the material solubilized by the solvents mixture. Fig. 10b shows a representative AFM image of the region where the polymer film was not yet removed, i.e. taken in the region on the right side in Fig. 10a. The film looks different from its initial state (for comparison, see Fig. 9b), showing that the bulk solvent mixture acted also on the regions not fully
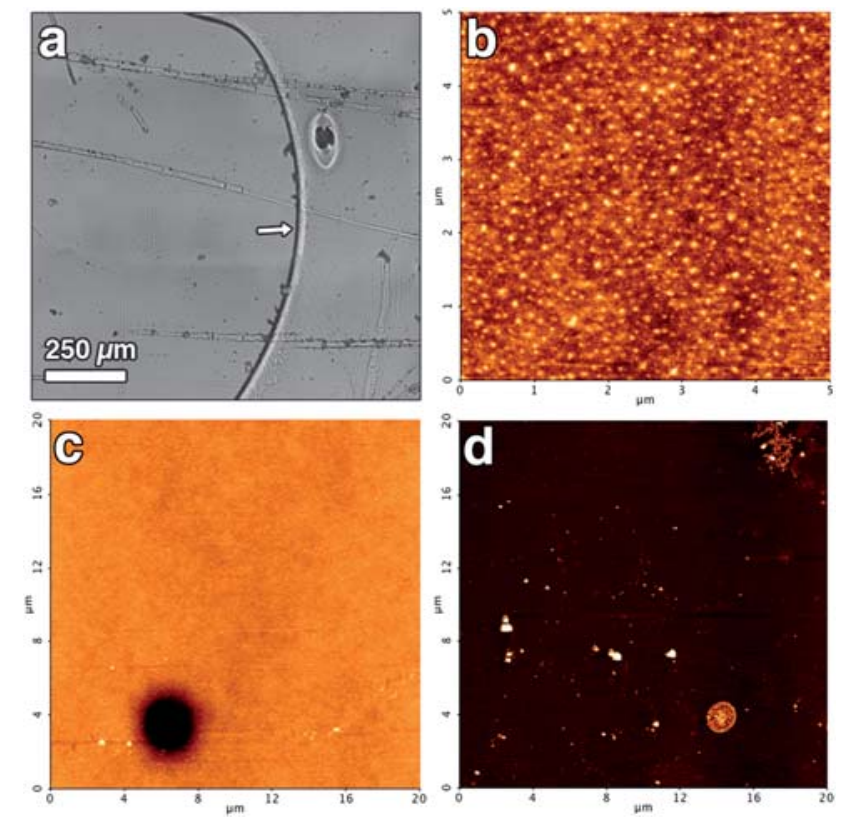

Fig. 10 (a) Optical micrograph of the Paraloid-coated mica slide after the cleaning treatment with EAPC without SDS. (b) AFM micrograph (height range: $5 \mathrm{~nm}$ ) of the same sample taken in the region on the right side of the optical micrograph. (c) Another AFM micrograph (height range: $15 \mathrm{~nm}$ ) in the same region of Fig. 10b showing a hole from where the cleaning process is believed to propagate. (d) AFM micrograph (height range: $15 \mathrm{~nm}$ ) of the same sample taken in the region on the left side of the optical micrograph. cleaned. In Fig. 10c we report an AFM image where a "hole" in the polymer film is visible. We believe that this "hole", reasonably resulting from a defect in the cast film, represents an initial stage in the formation of a larger region where the polymer would be removed in the case of a longer treatment. An AFM image taken inside one of these "clean" areas (i.e. in the region on the left side in Fig. 10a) is shown in Fig. 10d. Supporting the results obtained by optical microscopy, AFM confirms that, in the case of EAPC system without SDS, micro- and nanoscaled amorphous structures are present on the surface, resulting from the re-deposition of the material solubilized but not dispersed.

The role of the surface-active agent in the cleaning is clarified by the results of the experiment performed with the EAPC system with SDS. In Fig. 11a we report an optical microscopy image of a Paraloid-coated mica slide treated with such a system. The surface is optically homogeneous, as in the case of the film before the treatment, showing that the cleaning takes place in a uniform manner in the length scale accessible by optical microscopy. When the sample is investigated down to the nanoscale, AFM reveals that the polymer has not been homogeneously removed, after $10 \mathrm{~s}$ application. Fig. 11b shows a close-up of the film after the treatment: micro-scaled regions where the removal of the polymer proceeded deeper in the film are separated from each other by polymeric borders, with height of about 20 nanometres. An AFM image at lower magnification is shown in Fig. 11c, where it is possible to extract the size and the shape of the regions that were wet by the solvents to solubilize the polymer. These regions are schematically highlighted in Fig. 11d. Interesting enough, no re-deposited polymer is present inside the
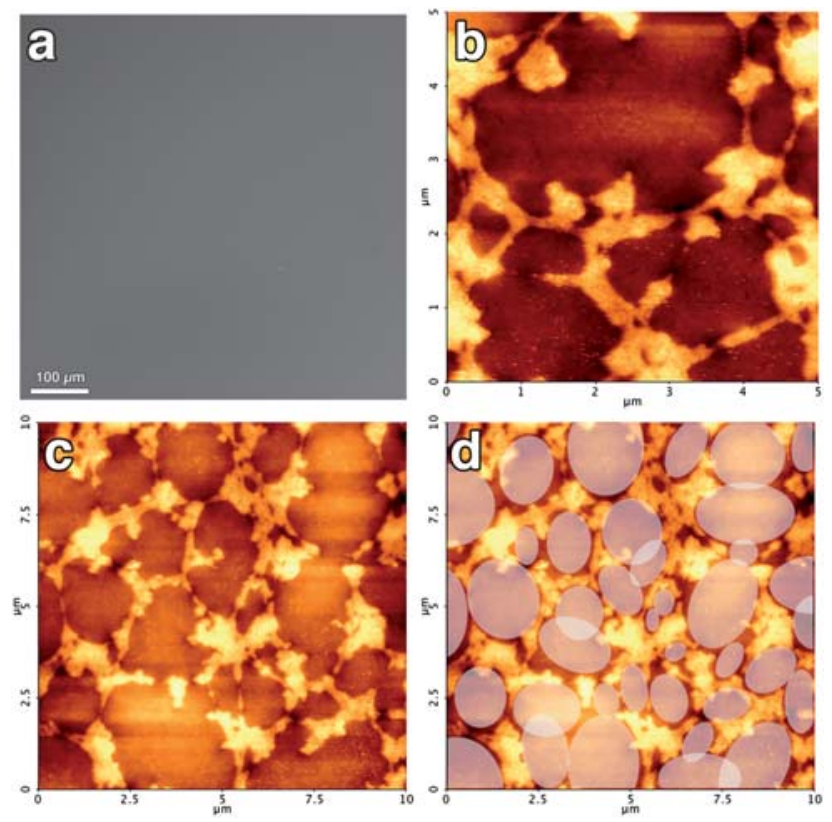

Fig. 11 (a) Optical micrograph of the Paraloid-coated mica slide after the cleaning treatment with the EAPC system (with SDS). (b) AFM micrograph (height range: $25 \mathrm{~nm}$ ) of the same sample showing the clean regions together with the polymer borders. (c) AFM micrograph (height range: $35 \mathrm{~nm}$ ) at lower magnification next to the same micrograph where the regions wetted by the solvents to solubilize the polymer are schematically highlighted (d). 

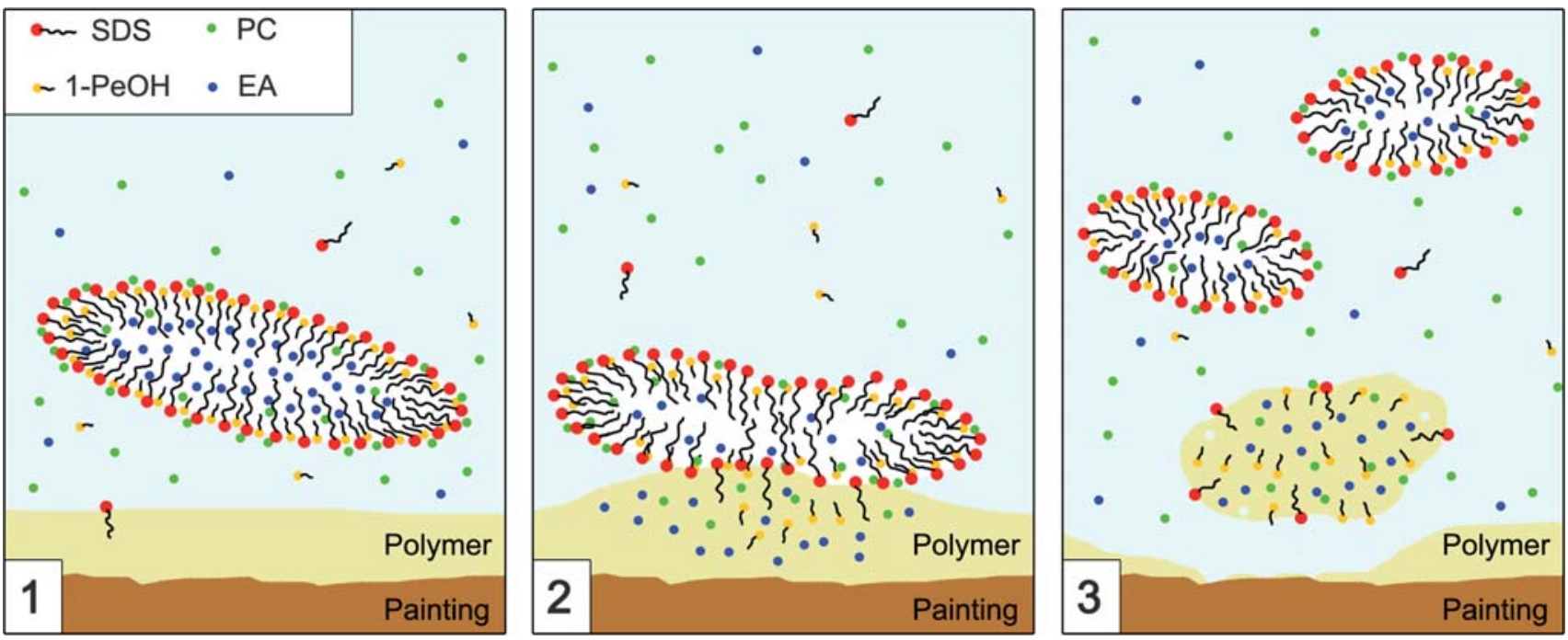

Fig. 12 Schematic representation of the mechanism of interaction between the micelles of the EAPC system and the polymer coating. (1) The micelle acts as solvent carrier and drives the interaction with the polymer. (2) The polymer absorbs the solvent mixture with the optimal properties (i.e. polarity and dispersion forces) to initiate a swelling process. (3) Due to the depletion in the organic phase, the micelles re-organize their structure and get smaller.

clean regions. The comparison of these results with those obtained without SDS prove the two-fold role of the surfactant: first, it confines the wetting in a micro-scaled area (while the wetting process is not controlled when no SDS is used); second, it helps in the dispersion of the removed polymer, avoiding its redeposition. Still, on the molecular scale the removal of the Paraloid film does not take place homogeneously, suggesting that the size of the wetting areas found by AFM could be an important parameter to pursue in optimizing our formulation.

For sufficiently longer application times, the EAPC system leads to the complete cleaning of the surface, while the EAPC without SDS experiences re-deposition of the polymer, this last effect is dramatically enhanced by the presence of surface inhomogeneities and porosity.

\section{Conclusions}

This study reports on a novel "swollen micellar system" (EAPC), successfully applied in the removal of acrylic and vinyl/acrylic copolymers from Mesoamerican wall paintings.

The EAPC system is the evolution of previous microemulsive or micellar systems containing propylene carbonate ${ }^{8-13,29,30}$ whose composition included the presence of PC or was based on water, SDS, 1-PeOH and PC. Ethyl acetate was added to this last system almost up to the maximum amount that micelles could uptake.

The main purpose of this contribution was the study of the interaction mechanism between the nanofluid and Paraloid B72 coatings, to investigate whether the detergency paradigm applies to explain the successful removal of polymer films from solid surfaces.

The EAPC nanofluid interacts in three distinct ways (as outlined in Fig. 12): (1) micelles provide the solvent blend reservoir, from which (2) the polymer film extracts the optimal composition that leads to its swelling and (3) the swollen polymer detaches from the substrate while the micelles get smaller and re-organize their structure due to the outflow of the solvents. It is worth pointing out some important features in this picture. First of all, the role of the micelles is crucial; from the comparison between the performances of the EAPC system and the same composition without SDS, it is clear that a nanocompartmentalized structure is required to perform successful polymer removal in an aqueous medium. Secondly, the mixture of solvents absorbed by the polymer for the swelling process is chosen by the polymer itself according to its physico-chemical characteristics. For this reason it is clear that a cleaning system should be able to provide the polymer the optimum solvent mixture to perform a good removal. A detailed description of the structural features of the EAPC system obtained through a SANS investigation will be reported in a forthcoming paper. ${ }^{22}$ We believe that a deep comprehension of all these factors is the key for the formulation of specifically tailored systems to face up to new conservative challenges.

\section{Acknowledgements}

The authors would like to thank Josè Texeira for his help during the SANS measurements and for his constant support during the data reduction; Carla Giovannone, Tiziana Dell'Omo, and Lucia Di Paolo (ISCR, Rome) for the realization of the wall painting model; Alfonso de Maria y Campos (director of INAH-Mexico), Lilia Rivero Weber (director of CNCPC-Mexico), Maria Del Carmen Castro and Yareli Jaidar Benavides (CNCPC-Mexico) for experiments in Cholula. A special thank to Maricarmen Casas and Diana Magaloni for introducing us to the Mesoamerican conservation issues. CSGI and MIUR are acknowledged for financial support. The access to the LLB neutron facility has been supported by the European Commission under the $7^{\text {th }}$ Framework Program: Integrated Infrastructure Initiative for Neutron Scattering and Muon Spectroscopy: NMI3/FP7contract no 226507. 


\section{Notes and references}

1 E. Carretti and L. Dei, Prog. Org. Coat., 2004, 49, 282-289.

2 Resins in Conservation, in Proceedings of the Symposium, Edinburgh, 21 and 22 May 1982, ed. J. O. Tate and N. H. Tennent, Scottish Society for Conservation and Restoration, Edinburgh, 1983.

3 C. V. Horie, Materials for Conservation, Butterworth, London, 1987.

4 R. L. Feller, Accelerated Aging: Photochemical and Thermal Aspects, D. Berland Ed., The Getty Conservation Institute, Los Angeles, 1994. 5 M. Lazzari and O. Chiantore, Polymer, 2000, 41, 6447-6455.

6 O. Chiantore and M. Lazzari, Polymer, 2001, 42, 17-27.

7 M. Favaro, R. Mendichi, F. Ossola, U. Rosso, S. Simon, P. Tomasin and P. A. Vigato, Polym. Degrad. Stab., 2006, 91, 3083-3096.

8 E. Carretti, L. Dei, C. Miliani and P. Baglioni, Progr. Colloid Polym. Sci., 2001, 118, 63-67.

9 E. Carretti, L. Dei and P. Baglioni, Langmuir, 2003, 19, 7867-7872.

10 E. Carretti, R. Giorgi, D. Berti and P. Baglioni, Langmuir, 2007, 23, 6396-6403.

11 S. Grassi, E. Carretti, P. Pecorelli, F. Iacopini, P. Baglioni and L. Dei, J. Cult. Heritage, 2007, 8, 119-125.

12 R. Giorgi, M. Baglioni, D. Berti and P. Baglioni, Acc. Chem. Res., 2010, 43(6), 695-704

13 E. Carretti, E. Fratini, D. Berti, L. Dei and P. Baglioni, Angew. Chem., Int. Ed., 2009, 47, 8966-8969.

$14 \mathrm{H}$. Orea and V. A. Magar, Preprints of the $13^{\text {th }}$ Triennial Meeting ICOM Committee for Conservation, ICOM-CC, Rio de Janeiro, 2227 September 2002, p. 176.

15 J. Riederer, Proceedings of $7^{\text {th }}$ Triennial ICOM Meeting, ICOM-CC, Copenhagen, Paris 1, 10-14 September 1984, 1984, p. 21.

16 A. Espinosa, Proceedings of in situ Archaeological Conservation, 6-13 April 1986, ed. H. W. M. Hodges, INAH, Mexico City, 1987, p. 84.
17 H. Toraya, C. R. Hubbard, S. B. Robie, D. Louer, H. E. Gobel, G. Will, R. Gilles, T. N. Blanton, T. C. Huang and T. Raftery, Powder Diffr., 1995, 10, 91-95.

18 G. Palazzo, D. Fiorentino, G. Colafemmina, A. Ceglie, E. Carretti, L. Dei and P. Baglioni, Langmuir, 2005, 21, 6717-6725.

19 G. Colafemmina, D. Fiorentino, A. Ceglie, E. Carretti, E. Fratini, L. Dei, P. Baglioni and G. Palazzo, J. Phys. Chem. B, 2007, 111, 7184-7193.

20 C. Stubenrauch, Microemulsions-Background, New Concepts, Applications, Perspectives, Wiley-Blackwell, 2008.

21 B. Cabane, Small Angle Scattering Methods, in Surfactant Solutions New Methods of Investigation, ed. R. Zana, Marcel Dekker, Inc., New York, 1987; Neutron, X-Ray and Light Scattering, ed. P. Lindner and Th. Zemb, North Holland, Amsterdam, 1991.

22 M. Baglioni, R. Giorgi, J. Teixeira, D. Berti and P. Baglioni, in preparation.

23 B. Bockstahl and G. Duplatre, J. Phys. Chem. B, 2001, 105, 1318.

24 E. Caponetti, D. Chillura Martino, M. A. Floriano and R. Triolo, Langmuir, 1997, 13, 3277-3283.

25 K. L. Thimons, L. C. Bradzill, D. Harrison and M. R. Fisch, J. Phys. Chem. B, 1997, 101, 11087-11091.

26 E. Y. Sheu and S. H. Chen, J. Phys. Chem., 1988, 92, 4466-4474.

27 S. H. Chen, E. Y. Sheu, J. Kalus and H. Hoffman, J. Appl. Crystallogr., 1988, 21, 751-769.

28 P. Samori, Scanning Probe Microscopies Beyond ImagingManipulation of Molecules and Nanostructures, Wiley-VCH, 2006.

29 H.-D. Dorfler and C. Swaboda, Colloid Polym. Sci., 1993, 271, 586599.

30 R. Ivanova, B. Lindman and P. Alexandridis, Langmuir, 2000, 16, 9058-9069. 